\title{
Simulation of surface pressure fluctuations on an Airbus-A320 fuselage at cruise conditions
}

\author{
$\mathrm{Nan} \mathrm{Hu}^{*}$, Christina Appel ${ }^{\dagger}$ \\ German Aerospace Center (DLR), 38108 Braunschweig, Germany \\ Stefan Haxter ${ }^{\dagger}$ \\ German Aerospace Center (DLR), 37073 Göttingen, Germany \\ Sören Callsen ${ }^{\S}$, Alexander Klabes ${ }^{\mathrm{I}}$ \\ Airbus Operations GmbH, 21129 Hamburg, Germany
}

The fuselage surface pressure fluctuations on an Airbus-A320 aircraft at cruise conditions are simulated by solving a Poisson equation. The right-hand-side source terms of the Poisson equation, including both the mean-shear term and the turbulence-turbulence term, are realized with synthetic turbulence generated by the Fast Random Particle-Mesh Method. The stochastic realization is based on time-averaged turbulence statistics derived from a RANS simulation under the same condition as in the flight tests, conducted with DLR's Airbus-A320 research aircraft. The fuselage surface pressure fluctuations are calculated at three streamwise positions from front to rear corresponding to the measurement positions in the flight tests. Features of the pressure fluctuations relevant to the fuselage surface excitation are obtained and analyzed. A good agreement for one- and two-point spectral characteristics of the surface pressure fluctuations is found between the simulation and flight tests results.

\section{Nomenclature}

$$
\begin{array}{lll}
\alpha & =\text { angle } \\
\beta_{\delta^{*}} & =\text { Clauser parameter } \\
\delta & =\text { boundary layer thickness, Dirac delta function } \\
\delta^{*} & =\text { boundary layer displacement thickness } \\
\delta_{i j} & =\text { Kronecker symbol } \\
\epsilon_{i j k} & =\text { Levi-Civita symbol } \\
\gamma & =\text { length-scale stretching factor } \\
\Gamma_{p p} & =\text { pressure coherence }
\end{array}
$$

\footnotetext{
Presented as Paper 2019-2728 at the 25th AIAA/CEAS Aeroacoustics Conference, Delft, The Netherlands, May 20-23, 2019

*Research Engineer, Department Technical Acoustics, nan.hu@dlr.de

$\dagger$ Research Engineer, Department Technical Acoustics, christina.appel@dlr.de

${ }^{\ddagger}$ Research Engineer, Department Experimental Methods, stefan.haxter@dlr.de

$\S$ Acoustic Engineer, soeren.callsen@airbus.com

ILoads \& Aeroelastics Engineer, alexander.klabes@ airbus.com
} 


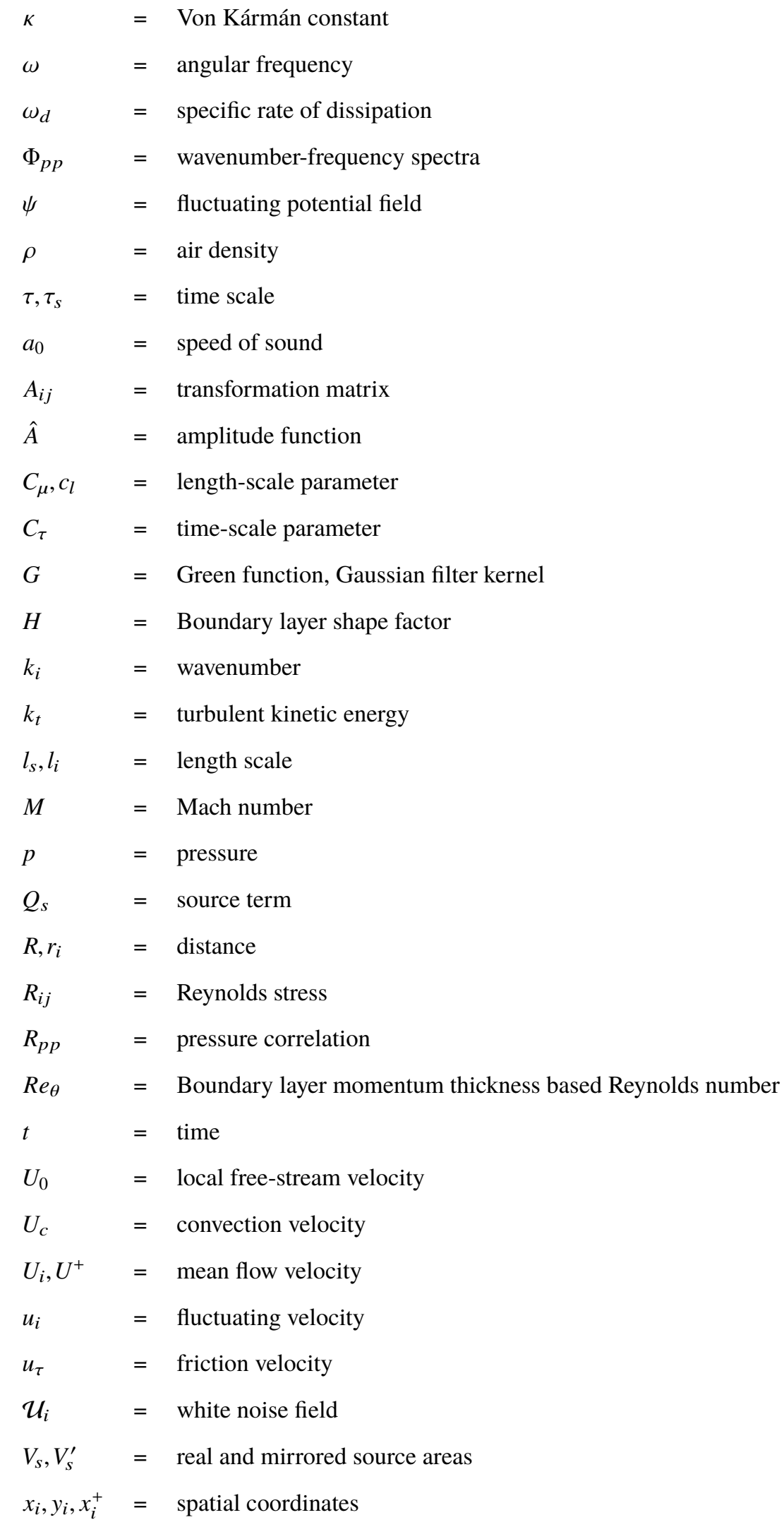




\section{Introduction}

With the reduction of jet noise through the development of high bypass ratio engines, noise induced by surface pressure fluctuations beneath turbulent boundary layers has become a major source for aircraft cabin noise in cruise flight [1]. The surface pressure fluctuations excite the fuselage, and consequently, the induced fuselage vibration radiates noise further into the cabin. Since cabin noise belongs to one of the decisive conditions for the airline passenger comfort, prediction and reduction of the boundary layer induced surface pressure fluctuations become more involved in the aircraft design process. To achieve a low-noise design, a fundamental understanding of this phenomenon and validated design tools for cruise conditions are required.

The knowledge on surface pressure fluctuations gained over the past decades is mostly restricted to incompressible flows [2--9]. Only a few studies were carried out on compressible flows, due to the limitations of test facilities or the expensive experimental/computational cost. Ehrenfried \& Koop [10] measured the surface pressure spectra beneath transonic boundary layers in the Transonic-Wind-Tunnel Göttingen (TWG). A problem faced by measuring in wind tunnels at high flow velocity is the high background noise level, which contaminates the measured pressure spectra up to $2 \mathrm{kHz}$ [10]. Numerical investigations on high-speed flows, however, only for low Reynolds number flows were carried out by Gloerfelt \& Berland [11], Duan et al. [12] and Cohen \& Gloerfelt [13]. Results of in-flight tests were published by Bhat [14], Palumbo [15, 16], Haxter \& Spehr [17, 18] and Klabes et al. [19]. In the most recent flight tests performed with DLR's Airbus-A320 Advanced Technology Research Aircraft (ATRA) [20], a pressure transducer array with a quasi-randomized distribution was applied in the flight tests. With array beamforming techniques, comprehensive features of the surface pressure fluctuations including wavenumber-frequency spectra were obtained. However, the corresponding boundary layer parameters are still lacking, and furthermore, such a boundary layer flow measurement in flight is hard to perform.

Due to the extremely high cost of the flight tests and the difficulties of measuring the boundary layer flow in flight, numerical applications in transonic boundary layer flows or directly performed on aircrafts at cruise conditions are strongly desired. Numerical methods based on resolving (fully or partially) wall-bounded turbulence for practical design applications on the high Reynolds number flows, such as in cruise conditions, are currently impossible to apply due to the extremely high computational cost. Therefore, an efficient numerical method, which can represent the most important features of the surface pressure field relevant to induced cabin noise, is of particular interest. Hu et al. [21] simulated the surface pressure fluctuations using a stochastic turbulence model, which enables a simulation at high Reynolds numbers. The fluctuating pressure within the boundary layer is calculated by solving a Poisson equation. Synthetic turbulence generated with the Fast Random Particle-Mesh Method (FRPM) [22] is used to describe the source terms on the right-hand side of the equation.

In the present work, the fuselage surface pressure fluctuations of an Airbus-A320 aircraft at cruise conditions are simulated using the method applied in Hu et al. [21]. Actually, the Poisson equation used in their method cannot govern 
the acoustic pressure induced by the compressible boundary layer flows. However, results from the flight tests [23] showed that the acoustic contribution to the surface pressure fluctuations is negligible compared to the hydrodynamic contribution. A discussion of this topic along with a description of the numerical approach is given in section II The computational setups for RANS and FRPM simulations are provided in section [II] Relevant features of the surface pressure field such as one-point spectra, coherence lengths and convection velocities are analyzed and compared to the flight test results in section IV

\section{Numerical approach}

\section{A. Poisson equation}

To calculate the surface pressure fluctuations, a Poisson equation which governs the fluctuating pressures in incompressible flows is solved. The Poisson equation reads

$$
\Delta p=-\rho\left(2 \frac{\partial U_{i} u_{j}}{\partial x_{i} \partial x_{j}}+\frac{\partial^{2}\left(u_{i} u_{j}-\overline{u_{i} u_{j}}\right)}{\partial x_{i} \partial x_{j}}\right),
$$

where $U_{i}$ denotes the mean flow velocity and $u_{i}$ the fluctuating velocity; $p$ is the fluctuating pressure and $\rho$ is the air density. The source term is on the right-hand side of the equation and comprises two parts. The first part is the mean-shear term and the second part is the turbulence-turbulence term.

For most applications, $U_{1} \gg U_{2,3}$, where $x_{1}$ denotes the streamwise direction, $x_{2}$ the wall-normal direction and $x_{3}$ the spanwise direction. Together with the condition $\partial U_{1} / \partial x_{2} \gg \partial U_{1} / \partial x_{1,3}$ and $\partial U_{i} / \partial x_{i}=0$ for incompressible flows, the Poisson equation becomes

$$
\Delta p=-\rho\left(2 \frac{\partial U_{1}}{\partial x_{2}} \frac{\partial u_{2}}{\partial x_{1}}+\frac{\partial^{2}\left(u_{i} u_{j}-\overline{u_{i} u_{j}}\right)}{\partial x_{i} \partial x_{j}}\right) .
$$

Note that, in some special cases the condition $U_{1} \gg U_{2,3}$ could not be met, e.g. the fuselage boundary layer flow in the vicinity of the wing, where the flow is deflected due to the presence of the wing. Therefore, the transverse flow component $U_{3}$ cannot be neglected. Furthermore, for regions where the flow has strong acceleration/deceleration, e.g. in the cockpit region, the contribution from the source term $\partial U_{1} / \partial x_{1}$ should not be neglected. Therefore, the following formulation of the Poisson equation for the calculation of the fuselage boundary layer flow is used:

$$
\Delta p=-\rho\left(2\left(\frac{\partial U_{1}}{\partial x_{2}} \frac{\partial u_{2}}{\partial x_{1}}+\frac{\partial U_{3}}{\partial x_{2}} \frac{\partial u_{2}}{\partial x_{3}}+\frac{\partial U_{1}}{\partial x_{1}} \frac{\partial u_{1}}{\partial x_{1}}\right)+\frac{\partial^{2}\left(u_{i} u_{j}-\overline{u_{i} u_{j}}\right)}{\partial x_{i} \partial x_{j}}\right)
$$

If we deal with a boundary layer flow on a solid surface, the fluctuating pressure can be calculated from the convolution 
of a free-space Green function with the right-hand side source terms including the mirrored part from the wall, i.e.

$$
\begin{aligned}
p(\mathbf{x}, t) & =\int_{\mathbf{V}_{\mathbf{s}}+\mathbf{V}_{\mathbf{s}}^{\prime}} Q_{s}(\mathbf{y}, t) \cdot G(\mathbf{x}-\mathbf{y}) d^{3} \mathbf{y}, \\
& =-\int_{\mathbf{V}_{\mathbf{s}}+\mathbf{V}_{\mathbf{s}}^{\prime}} \frac{Q_{s}(\mathbf{y}, t)}{4 \pi R} d^{3} \mathbf{y},
\end{aligned}
$$

where $Q_{s}$ denotes the right-hand side source terms in Eq. (3), $V_{s}$ and $V_{s}^{\prime}$ represent the real and mirrored source areas, respectively. The Green function for the Poisson equation is $G=-1 / 4 \pi R$, where $R=|\mathbf{x}-\mathbf{y}|$.

In compressible flows, such as in cruise flight conditions, the flow compressibility needs to be taken into account. The pressure fluctuations within a compressible boundary layer flow are governed by the convected wave equation

$$
\Delta p-\frac{1}{a_{0}^{2}} \frac{D^{2} p}{D t^{2}}=Q_{s}
$$

where $D / D t=\partial / \partial t+\mathbf{U} \cdot \nabla$ and $a_{0}$ is the speed of sound in the medium. This equation can be solved in the same manner as the Poisson equation,

$$
p(\mathbf{x}, t)=-\int_{\mathbf{V}_{\mathbf{s}}+\mathbf{V}_{\mathbf{s}}^{\prime}} \frac{Q_{s}\left(\mathbf{y}, t-R / a_{0}\right)}{4 \pi R} d^{3} \mathbf{y}
$$

The Green function for the convected wave equation is $G=-\delta\left(\mathbf{y}, t-R / a_{0}\right) / 4 \pi R$, where $\delta$ is the Dirac delta function. Due to the flow convection, a retarded time $\tau=t-R / a_{0}$ is introduced in the Green function and the distance $R$ between the source position $\mathbf{y}$ and the receiver position $\mathbf{x}$ is modified by $R=\left|\mathbf{x}-\mathbf{y}-U_{1} \cdot(t-\tau)\right|=|\mathbf{x}-\mathbf{y}-M \cdot R|$, where $M=U_{1} / a_{0}$. Those differences in the Green function for the convected wave equation compared to the Poisson equation could influence the surface pressure fluctuations in compressible flows.

The surface pressure fluctuations measured from the flight tests [18, 19] showed similar features to those in incompressible flows. Furthermore, the turbulent boundary layer induced acoustic pressure was found out to be negligible compared with the hydrodynamic pressure fluctuations on the fuselage surface [23]. Therefore, it is supposed that the main feature of the fuselage surface pressure fluctuations in cruise conditions can be captured by solving the Poisson equation.

In the present work, pressure fluctuations are computed via Eq. [5], which is solved in the wavenumber domain by using the convolution theorem. For an accurate numerical solution with this approach, a modification as introduced by Hockney and Eastwood [24] is applied, which provides an exact realization of the free-space Green function in conjunction with a Fourier transform method on the finite domain.

\section{B. FRPM}

The source term in Eq. (3) is generated by FRPM [22]. FRPM uses averaged turbulence statistics provided by CFD results to synthesize turbulent velocity fluctuations. One and two-point statistics of the turbulence flow are realized. The 
basic idea is to generate a fluctuating vector potential $\psi_{i}$ with three components from a convolution of spatial white noise $\mathcal{U}_{i}$ with a spatial Gaussian filter kernel $G$,

$$
\psi_{i}(\mathbf{x}, t)=\int_{\mathbf{V}_{\mathbf{s}}} \hat{A}(\mathbf{x}) G\left(\mathbf{x}-\mathbf{x}^{\prime}\right) \mathcal{U}_{i}\left(\mathbf{x}^{\prime}, t\right) d^{3} \mathbf{x}^{\prime},
$$

with

$$
G\left(\mathbf{x}-\mathbf{x}^{\prime}\right)=\exp \left(-\frac{\pi}{2} \frac{\left|\mathbf{x}-\mathbf{x}^{\prime}\right|}{l_{s}^{2}}\right)
$$

where $\hat{A}$ denotes an amplitude function whose appropriate scaling yields the desired variance of $\psi_{i}, \mathbf{x}$ defines field coordinates of the vector potential and $\mathbf{x}^{\prime}$ defines white noise field coordinates. Furthermore, $l_{s}$ is an integral turbulence length scale determined from the Reynolds averaged Navier-Stokes (RANS) calculation,

$$
l_{s}=\frac{c_{l}}{C_{\mu}} \frac{\sqrt{k_{t}}}{\omega_{d}}
$$

where $k_{t}$ is the turbulent kinetic energy and $\omega_{d}$ is the specific rate of dissipation. The constant $C_{\mu}=0.09$ and $c_{l}$ is estimated to be 0.54 [25], so the pre-factor $c_{l} / C_{\mu} \simeq 6.0$. An anisotropy of the length scale can be realized by applying a stretching factor $\gamma$. It is assumed that the relationship $l_{s}=\left(l_{1} l_{2} l_{3}\right)^{1 / 3}$ and $1 / \gamma \cdot l_{1}=l_{2}=l_{3}$. In the present calculation, $\gamma$ is chosen to be 1.5, according to Hu et al. [21].

The fluctuating velocities can be obtained by taking the curl of the fluctuating potential field $\psi$,

$$
u_{i}=\epsilon_{i j k} \frac{\partial \psi_{k}}{\partial x_{j}}
$$

For sufficiently slow spatially changing length scale $l_{s}$ and amplitude $\hat{A}$ the derivatives of $\psi_{i}$ can be expressed through analytical derivatives of the Gaussian filter kernel,

$$
u_{i}(\mathbf{x}, t)=\int_{\mathbf{V}_{\mathbf{s}}} \hat{A}(\mathbf{x}) \epsilon_{i j k} \frac{\partial G\left(\mathbf{x}-\mathbf{x}^{\prime}\right)}{\partial x_{j}} \mathcal{U}_{k}\left(\mathbf{x}^{\prime}, t\right) d^{3} \mathbf{x}^{\prime}
$$

The white noise field $\mathcal{U}_{i}$ is defined in a Lagrangian frame moving at local flow velocity. Additional temporal turbulence decay can be modeled by a Langevin equation, which introduces the decorrelation in the two-points statistics. Altogether, the cross-correlation properties of the white noise is given by

$$
<\mathcal{U}_{i}\left(\mathbf{x}^{\prime}, t\right) \mathcal{U}_{j}\left(\mathbf{x}^{\prime}+\mathbf{r}, t+\tau\right)>=\delta(\mathbf{r}-\mathbf{U} \tau) \exp \left(-\frac{|\tau|}{\tau_{s}}\right) \delta_{i j}
$$

where the bracket means an ensemble average, $\delta_{i j}$ is the Kronecker symbol, $\delta(\mathbf{r}-\mathbf{U} \tau)$ describes a frozen turbulence flow moving with the flow velocity $\mathbf{U}$ and $\exp \left(-|\tau| / \tau_{s}\right)$ involves the turbulence decay, i.e. the spatially white noise is 
correlated in time with time-scale $\tau_{s}$. The local time scale is determined from RANS calculations,

$$
\tau_{s}=C_{\tau} \frac{l_{s}}{\sqrt{k_{t}}}
$$

The pre-factor $C_{\tau}=2.5$ is applied herein which is determined based on a comparison between the measured and calculated streamwise coherence lengths. The applied pre-factor is larger than $C_{\tau}=1.2$ used in incompressible flow cases, according to $\mathrm{Hu}$ et al. [21]. Based on Eqs. [13, 14], a larger value of $C_{\tau}$ slows the coherence decay. The streamwise coherence length increases by approximately $40 \%$ using the applied value of $C_{\tau}$ compared to the one used in the incompressible flows. One reason for the adjustment of the pre-factor may be the change in the Green function of the Poisson equation due to the flow convection, which is not covered by the present approach.

The Reynolds stress anisotropy can be obtained using a scaling tensor, which is derived from the relationship between the anisotropic Reynolds stress provided by the RANS calculation and the isotropic Reynolds stress tensors.

For more details about the FRPM implementation in computational domain and properties of the generated synthetic turbulence the reader is referred to Ewert et al. [22], and about the approach of the solution method and turbulence anisotropy referred to $\mathrm{Hu}$ et al. [21].

\section{Computational setups}

\section{A. CFD}

Mean flow statistics of the Airbus-A320 aircraft at cruise conditions are obtained from a RANS calculation with DLR's in-house CFD code TAU [26], applying a Reynolds Stress turbulence model. Transport equations with an additional equation for the length scale are solved for each component of the Reynolds stress. A formulation of $g\left(=\sqrt{1 / \omega_{d}}\right)$ is applied for the length-scale equation, where $\omega_{d}$ denotes the specific rate of dissipation. The use of the $g$ formulation can reduce the dependence on the near-wall mesh resolution [27], which could be an issue for complex geometries, e.g. the transition of the wing to the fuselage. Furthermore, upwind discretization for the inviscid fluxes and Runge-Kutta time stepping scheme are applied in the calculation.

For the aeroacoustics computation of the fuselage surface pressure fluctuations, a well-resolved boundary layer CFD solution is required. Following the Airbus best practices of mesh generation with the grid generator SOLAR [28], the fuselage itself is not sufficiently fine meshed for aeroacoustics-computation purposes. To achieve a sufficiently fine CFD mesh from the aeroacoustics-computation point of view, some simplifications of the aircraft geometry in terms of neglecting details are needed [29]. Therefore, all attachments at the wing, like winglets, flap tracks and engines, are removed to create a clean wing. The mesh contains about 17 million nodes in total, thereby 13 million nodes belong to hexahedral elements in the near field of the fuselage, see Fig. 1 (a). Thanks to the thick hexahedral layer, the boundary layer is well resolved and the value of the non-dimensional distance $y^{+}$from the wall to the first mesh node is $y^{+}<1$ all 


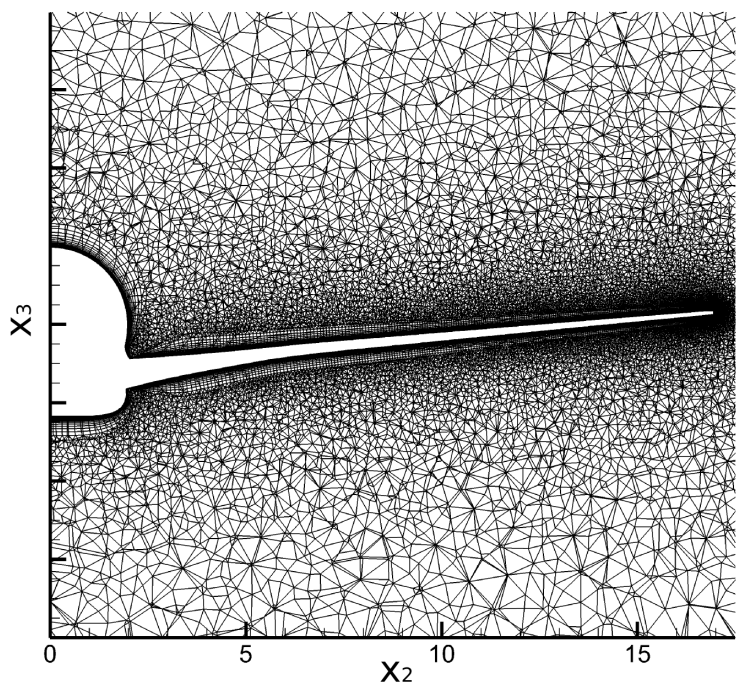

(a)

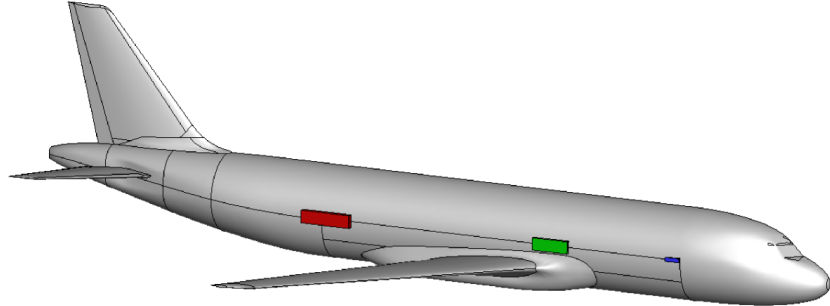

(b)

Fig. 1 (a) CFD mesh for the clean wing configuration; (b) FRPM computational domains.

over the fuselage.

\section{B. FRPM}

Despite the high efficiency of the applied numerical approach, the calculation of surface pressure fluctuations, especially for high-speed flows, is still a time and resources consuming numerical method compared to CFD calculations. Today, CFD calculations can be easily applied to full aircraft models and results are, thanks to high-performance computing, available within several days. Due to the relatively high computational cost of the surface pressure fluctuations calculation, the FRPM computational domain has to be limited to small areas. These computation areas correlate to the areas on the aircraft, where the surface pressure fluctuations were measured during three different flight test campaigns, refer to the colored meshes in Fig. 1. b). The computational area is larger towards the rear due to the increasing boundary layer thickness along the streamwise direction.

The mean flow and turbulence statistics obtained from the CFD calculation is interpolated into the FRPM mesh for the realization of synthetic turbulence. Instead of a Cartesian coordinate used in the CFD calculation, the FRPM mesh is built in a cylindrical coordinate. A transformation of the results from the Cartesian coordinate to the cylindrical coordinate is needed. In the cylindrical coordinate, the $x_{2}$ direction denotes the wall-normal direction and $x_{3}$ direction 
denotes the spanwise direction. The transformation is realized with the help of a transformation matrix, defined as

$$
A=\left(\begin{array}{ccc}
1 & 0 & 0 \\
0 & \cos \alpha & \sin \alpha \\
0 & -\sin \alpha & \cos \alpha
\end{array}\right),
$$

where $\alpha$ is the rotation angle between the coordinates. The transformation of the velocity vector $U_{i}$ and the Reynolds stress $R_{i j}$ is performed with

$$
\begin{aligned}
U_{i}^{(c y l)} & =\sum_{j=1}^{3} A_{i j} U_{j}^{(\text {cart })} \\
R_{i j}^{(c y l)} & =\sum_{k=1}^{3} \sum_{l=1}^{3} R_{k l}^{(c a r t)} A_{k i}^{-1} A_{l j}^{-1} .
\end{aligned}
$$

However, the currently applied numerical approach operates in a Cartesian coordinate system. To enable the simulation, the FRPM mesh generated in the cylindrical coordinate system has to be bent straight in the spanwise direction. This process may introduce an extra error in the calculation of the surface pressure fluctuations. For the calculation on the fuselage, due to the minor ratio of the boundary layer thickness and the fuselage curvature radius, this error is estimated to be negligible.

Calculations for one- and two-point statistics of the surface pressure are performed with two different FRPM mesh resolutions. For the one-point statistics calculation, a fine mesh resolution is used, aimed to achieve a good result of the one-point spectrum up to $5 \mathrm{kHz}$, which is considered as the relevant frequency range for aircraft cabin noise. The calculation is confined to a small domain, whose dimension is $3 \delta$ ( $\delta$ is the boundary layer thickness), $1.05 \delta$ and $2.5 \delta$ in the streamwise, wall-normal and spanwise directions, respectively. Furthermore, the applied Hockney's method, used to solve the Poisson equation, demands a mesh with $2^{N}$ points in each direction. A Cartesian mesh with $128 \times 64 \times 128$ points is chosen for the calculation of the one-point statistics. To achieve a comprehensive analysis of the two-point statistics, a considerably larger calculation domain is needed, whose dimension is extended to $12 \delta$ and $3 \delta$ in the streamwise and spanwise directions, respectively. At the mid position, a large misalignment (about $15^{\circ}$ ) between the flow direction and the FRPM mesh coordinate is produced due to the influence of the wing. Thus, a larger lateral area of $4 \delta$ is chosen. Furthermore, a relatively long time series database is required for the two-point statistics analysis. To keep the computational cost still relatively low for an industrial applicability, a rough Cartesian mesh with $256 \times 32 \times 64$ points is used for the calculation of the two-point statistics. The calculations are conducted on $8 \mathrm{CPU}$ threads. The benefit of parallel computing on even more CPU threads is small because parallel computing on the multi-FRPM patches for the applied approach is still under development. For the present calculations, $1.5 \mathrm{~s}$ 


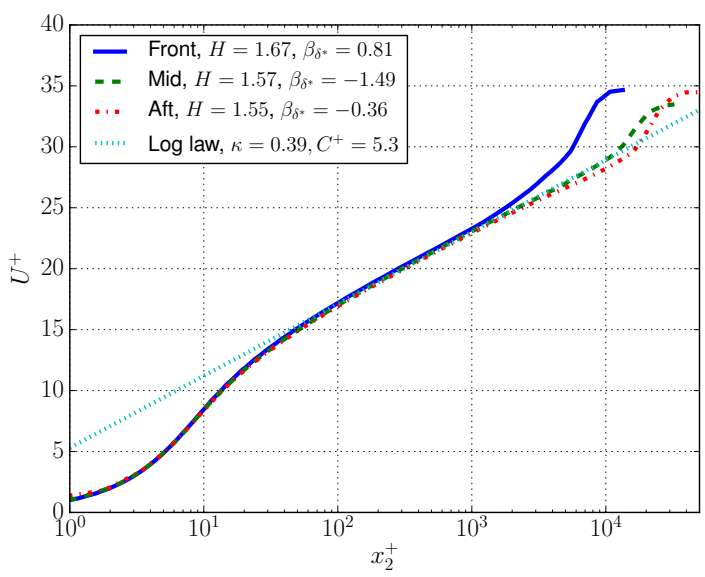

(a)

Fig. 2 Mean velocity profiles in wall units at different streamwise positions.

simulation time samples are computed for the two-point statistics analysis and $0.4 \mathrm{~s}$ for the one-point statistics analysis. The computation time for both cases is comparable, which is about 16 days for the front position and only 5 days for the aft position. The lower computation cost at the aft position is due to the larger computation time step benefit from the larger boundary layer thickness.

\section{Results}

\section{A. Mean velocity profiles and turbulence velocity fluctuations realization}

Fig. 2 shows the mean velocity profiles for the three calculated positions. The velocity profiles are comparable with that of an incompressible flow and agree with the log law by $\kappa=0.39$ and $C^{+}=5.3$. According to the Clauser parameter $\beta_{\delta^{*}}$, the front and aft positions of the calculation positions are subjected to small adverse and positive pressure gradients, respectively. At the mid position, a mild favorable pressure gradient is present. Note, that the boundary layer shape factor $H \geq 1.55$, which is considerably larger than expected in an incompressible flow. For the calculated positions with $40000<R e_{\theta}<120000$, the shape factor for a zero-pressure-gradient incompressible flow is estimated to be $1.26 \leq H \leq 1.3$ [30]. The larger shape factor in the present case is due to the decreasing air density towards the wall caused by the increasing temperature in a compressible boundary layer flow.

Turbulence velocity fluctuations realized by FRPM are used to prescribe the fluctuating source terms of Eq. (3). To verify a proper realization of the fluctuating velocity from FRPM, Fig. 3(a) shows the reconstructed Reynolds stress components in comparison to the initial value on the FRPM mesh from the RANS calculation. A very good reconstruction of all the Reynolds stress components for the outer region $>0.3 \delta$ is shown in Fig. 3 a) for both applied meshes. In the inner region, especially for $<0.1 \delta$, the realized Reynolds stresses drop dramatically compared to the RANS results. The main reason for this is that the mesh resolution is not sufficiently fine to realize the small turbulence 


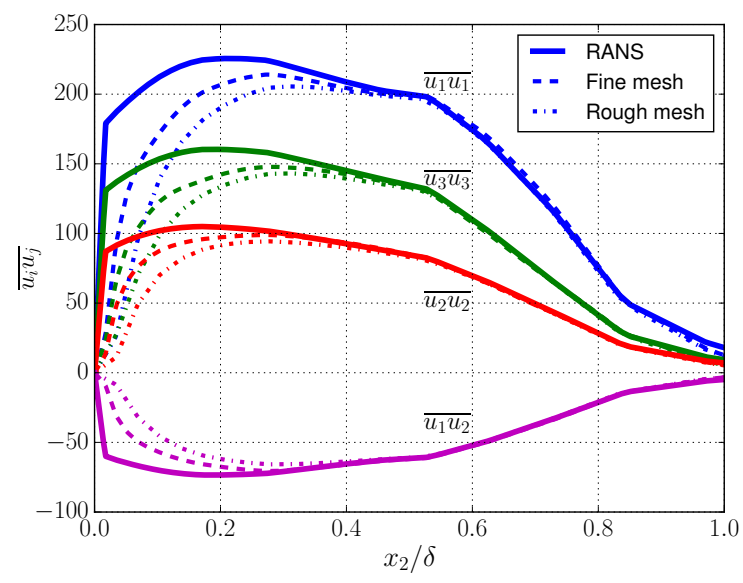

(a)

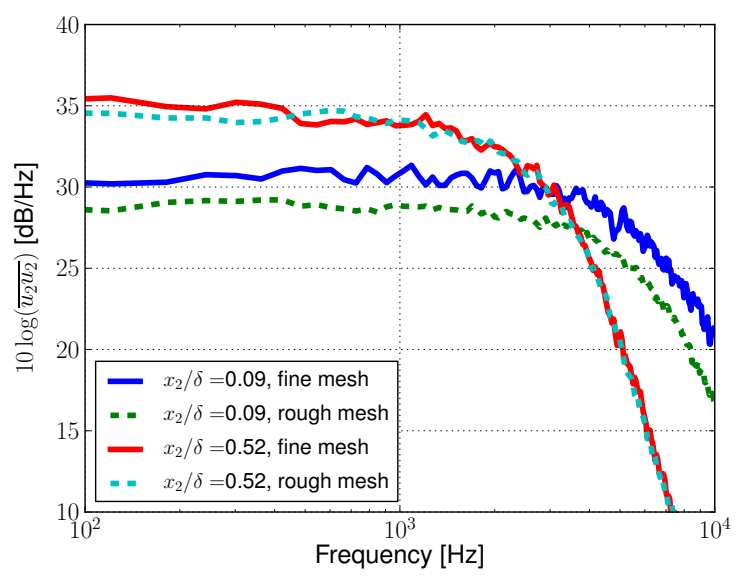

(b)

Fig. 3 Reconstruction of FRPM for the front position, (a) Reynolds stress components; (b) $u_{2}$ spectra.

structures in the near wall area and this issue is more severe for the rough mesh. Fig. 3 b) illustrates the difference between both meshes for the realized $u_{2}$ spectra. Due to the mesh resolution, a lower spectral level is shown for the rough mesh compared to the fine mesh at the position $x_{2}=0.09 \delta$.

\section{B. One-point spectra}

The pressure fluctuations are computed according to Eq. (4) using integration by parts. The mean-shear term $p_{m s}$ and the turbulence-turbulence term $p_{t t}$ are separately solved as

$$
\begin{aligned}
p_{m s}(\mathbf{x}, t)= & 2 \int_{\mathbf{V}_{\mathbf{s}}+\mathbf{V}_{\mathbf{s}}^{\prime}} \rho(\mathbf{y})\left(\frac{\partial U_{1}}{\partial x_{2}} u_{2}(\mathbf{y}, t) \cdot \frac{\partial G(\mathbf{x}-\mathbf{y})}{\partial x_{1}}+\frac{\partial U_{3}}{\partial x_{2}} u_{2}(\mathbf{y}, t) \cdot \frac{\partial G(\mathbf{x}-\mathbf{y})}{\partial x_{3}}\right. \\
& \left.+\frac{\partial U_{1}}{\partial x_{1}} u_{1}(\mathbf{y}, t) \cdot \frac{\partial G(\mathbf{x}-\mathbf{y})}{\partial x_{1}}\right) \mathrm{d}^{3} \mathbf{y}, \\
p_{t t}(\mathbf{x}, t)= & \int_{\mathbf{V}_{\mathbf{s}}+\mathbf{V}_{\mathbf{s}}^{\prime}} \frac{\partial \rho(\mathbf{y})\left(u_{i} u_{j}-\overline{u_{i} u_{j}}\right)(\mathbf{y}, t)}{\partial x_{i}} \cdot \frac{\partial G(\mathbf{x}-\mathbf{y})}{\partial x_{j}} \mathrm{~d}^{3} \mathbf{y} .
\end{aligned}
$$

Summing these two parts up, i.e. $p_{t o t a l}=p_{m s}+p_{t t}$, we obtain the pressure fluctuations within the boundary layers. In Eqs. $(18-19], \rho$ denotes the mean air density, and the density fluctuations are not considered because they are negligible compared to the mean air density for the considered Mach number (estimated to be approximately $1 \%$ ) according to Gerolymos and Vallet [31].

Fig. 4 shows the calculated power spectral density (PSD) for the surface pressure fluctuations at the front, mid and aft positions depicted with colors in Fig. 1]b). The spectra are computed using Welch's method [32] with a Hanning window, 512 samples per window, and 50\% overlap. The spectral levels are referenced to a $20 \mu$ Pa reference pressure. For all three positions, the numerical results show good agreement with the measured results [19], except for the 


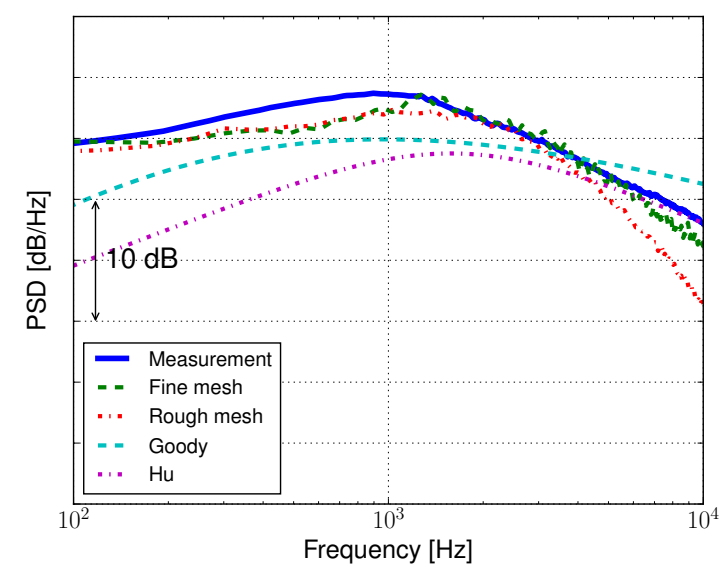

(a)

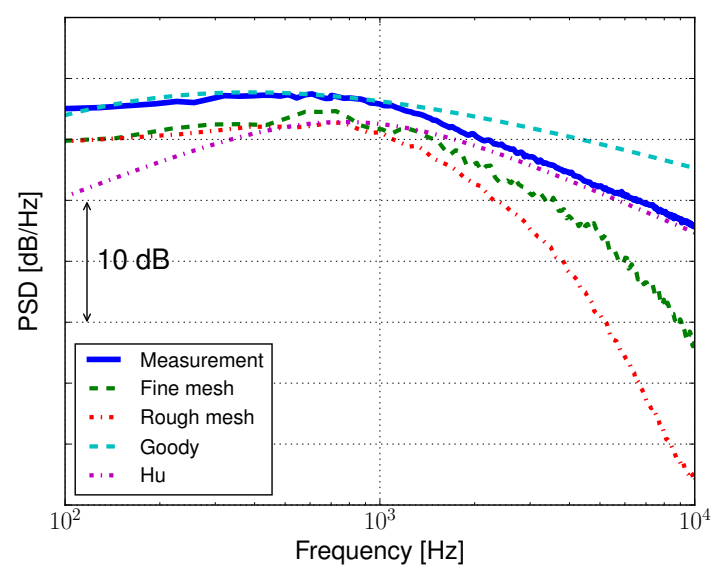

(b)

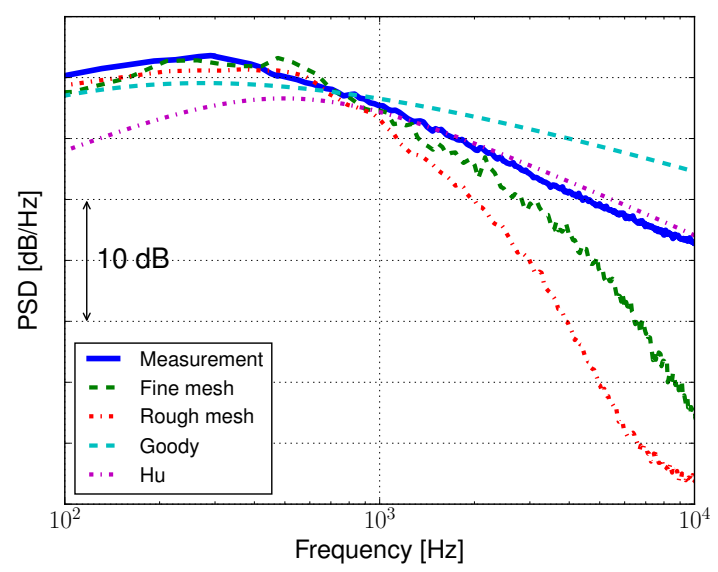

(c)

Fig. 4 One-point spectra at different streamwise positions, (a) front; (b) mid; (c) aft.

high-frequency spectra at mid and aft positions. The peak position moves towards lower frequencies as the measurement position moves towards the rear and the peak level at the aft position is the largest. These features are represented correctly by the numerical results.

The problem of the high-frequency attenuation by the numerical results is caused by the lack of Reynolds stress realization in the near wall area (refer to Fig. 3), where the small turbulence structures contribute mostly the highfrequency spectrum of the surface pressure fluctuations [21, 33, 34]. Therefore, the calculated spectra fall much faster at high frequencies compared to the measured spectra, and this problem is worse for the results calculated with the rough mesh. Because of the thicker boundary layer thickness at the aft position, the rapid drop-off region for the calculated spectrum begins earlier than the upstream positions. Nevertheless, the calculated spectrum at the aft position with the fine mesh shows good agreement with the measured spectrum up to $5 \mathrm{kHz}$, which is the upper frequency of interest to 
the industrial application.

According to analytical calculations for the mean-shear term $p_{m s}$ [21, 33, 34], the low-frequency surface pressure fluctuations are contributed by both the inner and outer boundary layer portions. The numerical studies by Kim [8], Hu et al. [21] showed that the surface pressure spectrum at low frequencies contributed by the turbulence-turbulence term is even larger than that by the mean-shear term. However, the contribution of the turbulence-turbulence term in the inner layer to the low-frequency spectrum is not clear to authors' knowledge. Nevertheless, the reduction of the realized turbulent fluctuations close to the wall, especially the component $\overline{u_{2} u_{2}}$ (the major source for the mean-shear term), may also lower the spectral magnitude at low frequencies. A slightly smaller magnitude of the calculated spectrum with the rough mesh can be observed at frequencies lower than $200 \mathrm{~Hz}$ for the front position compared to that calculated with the fine mesh, see Fig. $4($ a). Note, that the small discrepancy at low frequencies may also be attributed to the short time series database for the averaging process. Thus, additional calculations with a medium rough mesh (similar to the present case) and a very rough mesh are performed for the front position. The result is, for readability reasons, presented in Appendix $\mathrm{A}$, and shows a spectral attenuation at frequencies lower than $550 \mathrm{~Hz}$ for both rough meshes compared to the fine mesh. The attenuation is larger with increasing mesh roughness and reaches up to $2 \mathrm{~dB}$ for the result calculated with the roughest mesh.

Furthermore, empirical predictions of the one-point spectra are made using Goody's model [5] and Hu's model [7]. The Goody model is the most frequently used empirical model for zero-pressure-gradient flows, which shows fairly good predictions of the peak magnitude and position. However, the predicted spectra fall much slowly at high frequencies than the measured spectra, although the flows are subjected to small pressure gradients at the front and aft positions. The reason for the faster spectral roll-off is probably due to the decreasing air density towards the wall, which reduces the contribution of near-wall turbulence to the high-frequency wall pressure fluctuations, refer to Eqs. 18,19$]$. The Hu model introduced the boundary layer shape factor $H$ to predict the change of the high-frequency spectral slope caused by adverse pressure gradients. A larger shape factor of the boundary layer flow would give a prediction of a steeper slope. Fig. 4 shows an excellent prediction of the high-frequency spectral slope and magnitude by the Hu model. This may indicate that the shape factor can reflect the effect of the air density change on the high-frequency wall pressure spectrum. However, due to the imprecise prediction of the peak position, the Hu model underpredicts the peak magnitude.

\section{Coherence and wavenumber-frequency spectra}

The space-time correlation for the surface pressure fluctuations over $\left(x_{1}, x_{3}\right)$ plane is defined by,

$$
R_{p p}(\mathbf{x}, \mathbf{r}, \tau)=\frac{<p^{\prime}(\mathbf{x}, t) p^{\prime}(\mathbf{x}+\mathbf{r}, t+\tau)>}{\sqrt{<p^{\prime 2}(\mathbf{x}, t)><p^{\prime 2}(\mathbf{x}+\mathbf{r}, t+\tau)>}}
$$


where $\mathbf{x}=\left(x_{1}, x_{3}\right), \mathbf{r}=\left(r_{1}, r_{3}\right) . \quad R_{p p}(\mathbf{x}, \mathbf{r}, \tau)$ denotes the correlation at the surface point $\left(x_{1}, x_{3}\right)$. If the surface pressure fluctuations field can be treated as a homogeneous field, so the correlation is not location-dependent, $R_{p p}(\mathbf{x}, \mathbf{r}, \tau) \sim R_{p p}(\mathbf{r}, \tau)$. This assumption is well fulfilled for fully developed zero-pressure-gradient turbulent boundary layers. Thus, the coherence spectrum can be defined by

$$
\Gamma_{p p}\left(r_{1}, r_{3}, \omega\right)=\frac{1}{2 \pi} \int_{-\infty}^{\infty} R_{p p}\left(r_{1}, r_{3}, \tau\right) \exp (-i \omega \tau) \mathrm{d} \tau
$$

The surface pressure fluctuations needed for the coherence calculation are recorded with a rectangular microphone array with a dimension of $10 \delta$ and $1.5 \delta$ in the streamwise and spanwise directions, respectively. The corresponding resolution of the array is $2 \Delta x_{1}$ and $\Delta x_{3}$, where $\Delta x_{i}$ denotes the resolution of the FRPM mesh. The coherence is computed using Welch's method with a Hanning window, 256 samples per window, and 50\% overlap. Due to the short window length, an additional loss in coherence will be induced, especially at large streamwise separations, if the convection time of the surface pressure fluctuations is not taken into account [16]. Therefore, a time offset defined by $\mathbf{r} / U_{c}$ between two microphone positions is applied, where $U_{c}=0.8 U_{0}$ ( $U_{0}$ is the local free-stream velocity). The value of the convection velocity is estimated for the large separations, where this issue is mainly relevant. Coherence between each array point and the four corner points is calculated. The obtained four coherence maps are merged together.

As an example, Fig. 5 shows the absolute value of coherence at $2417 \mathrm{~Hz}$ for the front position, calculated in the larger computational domain with the rough mesh. The selected frequency is a coincidence frequency of the wavenumberfrequency spectra between the simulation and flight test results. Furthermore, the measured wavenumber-frequency spectrum at this frequency has a good signal-to-noise ratio and shows a clear convection ridge, refer to Fig. 6. b).

The result of the coherence shows an elongated pattern in the streamwise direction and an inclined angle to the $x_{1}$ coordinate. This angle denotes the misalignment between the flow direction and the fuselage longitudinal axis. A discontinuity in the obtained coherence between the positive and negative areas is observed at $x_{1}=0$, which is primarily caused by a weak inhomogeneity of the boundary layer flow and a slight change of the flow direction within the calculation area.

By taking the spatial Fourier transform of the coherence map, we obtain the wavenumber-frequency spectrum,

$$
\Phi_{p p}\left(k_{1}, k_{3}, \omega\right)=\frac{1}{(2 \pi)^{2}} \int_{-\infty}^{\infty} \int_{-\infty}^{\infty} \Gamma_{p p}\left(r_{1}, r_{3}, \omega\right) \exp \left(i k_{1} r_{1}\right) \exp \left(i k_{3} r_{3}\right) \mathrm{d} r_{1} \mathrm{~d} r_{3},
$$

where $k_{1,3}$ are the streamwise and spanwise wavenumbers. The signal used in the processing is restricted to an area of four times the size of the streamwise and spanwise coherence lengths. With the restricted signal area, the signal-to-noise ratio can be improved at high frequencies, where the coherence length is small and the array size is overly large for the signal processing [35]. The signal field is filtered with a 2-D Hanning window to avoid a step on the field border and to 


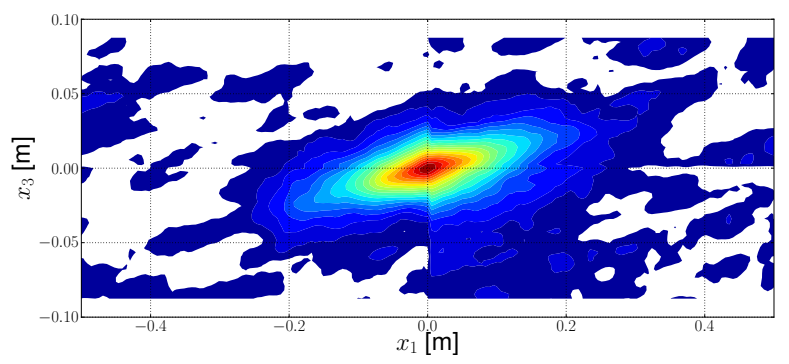

Fig. 5 Contour plots of calculated coherence $\left|\Gamma_{p p}\left(r_{1}, r_{3}, \omega\right)\right|$ at $2417 \mathrm{~Hz}$ for the front position, levels between 0.05 and 1 with increment of 0.05 .

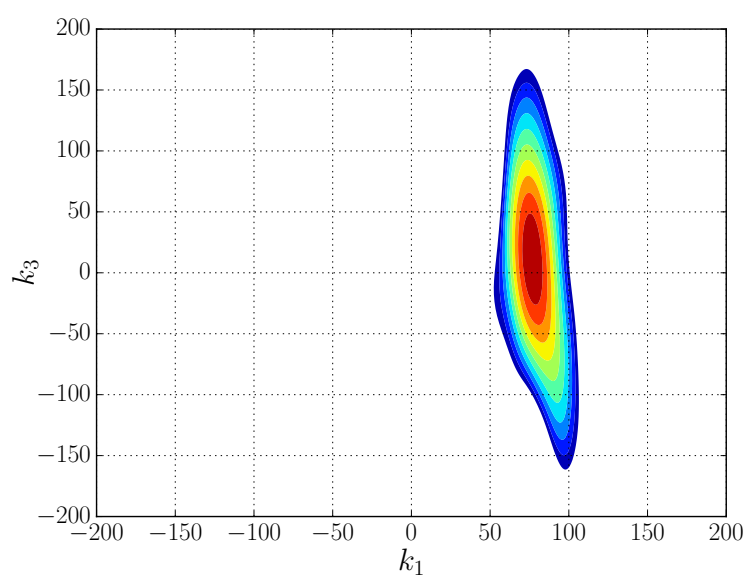

(a)

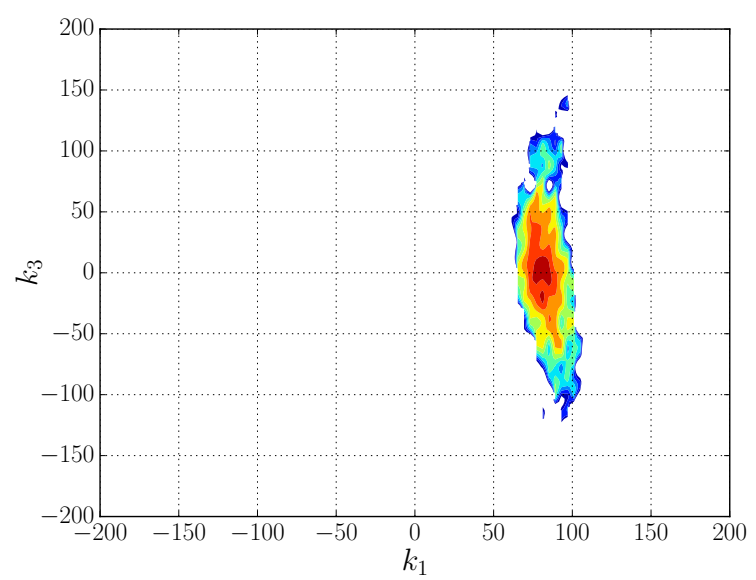

(b)

Fig. 6 Contour plots of wavenumber-frequency spectra $\Phi_{p p}\left(k_{1}, k_{3}, \omega\right)$ at $2417 \mathrm{~Hz}$ for the front position, levels between $-10 \mathrm{~dB}$ and $0 \mathrm{~dB}$ with increment of $1 \mathrm{~dB}$, (a) simulation; (b) measurement.

reduce the importance of the border area where the signal-to-noise ratio is low. Furthermore, to achieve a fine resolution of the wavenumber-frequency spectrum, the array size is extended six times with zero-padding.

Fig. 6shows the obtained wavenumber-frequency spectrum, which is in good agreement with the measured spectrum [18]. The shape and position of the convection ridge are well predicted. Note, that both spectra are referred to their respective peak values, which have a difference of $2.5 \mathrm{~dB}$. Besides uncertainties of the measurement and simulation, the difference in the absolute level of the wavenumber-frequency spectra can be caused by the different signal-to-noise ratio, microphone array geometries and array processing methods.

\section{Parameters for two-point statistics}

Corcos [4] found that the streamwise and spanwise coherence of the surface pressure fluctuations can be well represented by exponential functions with appropriate decay rates. Using the coherence length and the convection velocity to predict the coherence decay and the streamwise phase difference, Corcos proposed a rhombic shaped 
coherence model, expressed as

$$
\Gamma_{p p, \text { rhombic }}\left(r_{1}, r_{3}, \omega\right)=\exp \left(-\left|r_{1}\right| / l_{1}\right) \exp \left(-\left|r_{3}\right| / l_{3}\right) \exp \left(i \omega r_{1} / U_{c}\right)
$$

where $r_{1,3}$ are the separation in the streamwise and spanwise directions, $l_{1,3}$ are the coherence lengths in both directions, $U_{c}$ is the convection velocity and $\omega$ is the angular frequency. Smol'yakov and Tkachenko [36] used the same function to formulate the streamwise and spanwise coherence, but an elliptic form to formulate the off-axis coherence,

$$
\Gamma_{p p, \text { elliptic }}\left(r_{1}, r_{3}, \omega\right)=\exp \left(-\sqrt{\left(r_{1} / l_{1}\right)^{2}+\left(r_{3} / l_{3}\right)^{2}}\right) \exp \left(i \omega r_{1} / U_{c}\right)
$$

If the flow direction is not aligned with the applied coordinate system, an inclination of the coherence pattern would be caused, e.g. see Fig. 5. For those cases, the separation in the flow streamwise direction should be redefined as $r_{1}=\cos \alpha \cdot \tilde{r}_{1}+\sin \alpha \cdot \tilde{r}_{3}$ and in the flow spanwise direction $r_{3}=-\sin \alpha \cdot \tilde{r}_{1}+\cos \alpha \cdot \tilde{r}_{3}$, where $\tilde{r}_{1,3}$ denotes the longitudinal and lateral separations and $\alpha$ denotes the convection angle referring to the applied coordinate system.

Therefore, the coherence lengths, the convection velocity and the flow direction are the important parameters, with which the coherence of the surface pressure fluctuations can be formulated. Those parameters are calculated based on the obtained coherence pattern and wavenumber-frequency spectra in section IV.C. The results are compared to the results obtained from the flight tests [18, 37]. Both simulation and measurement results shown in this section are calculated with the same procedure.

Firstly, the flow direction is determined based on the coherence pattern, refer to Fig. 5. For each frequency, a linear function is fitted through the points, at which the coherence is greater than $\exp (-1) \simeq 0.367$. The resulted deviation of the linear function from the the array longitudinal axis (aircraft fuselage axis) corresponds to the flow convection angle.

Fig. 7 shows the obtained convection angles for the calculated positions. Good agreement between the simulation and measurement results for all three positions is shown. The scatter of the measurement results is larger than the simulation results due to a lower signal-to-noise ratio. The scatter of the simulation result at the aft position increases at frequencies larger than $4.5 \mathrm{kHz}$ (not shown). The reason is probably that the signal level is too low at high frequencies for the aft position, refer to Fig. 4(c) for the rough mesh, which lowers the signal-to-noise ratio. It is worth noting that, for the aft position, the measurement results are derived from the idle flight condition (averaged velocity equal to the cruise flight velocity) because the surface pressure fluctuations at the aft position are also contributed by jet noise at cruise conditions.

Fig. 7 shows a small positive convection angle at the front position. This indicates that the flow direction has a small positive inclination against the fuselage longitudinal axis (aircraft pitch angle is $2^{\circ}$ for the calculation case). The inclination increases at the mid position due to the influence of the wing, refer to Fig. 1. (b), and the convection angle 


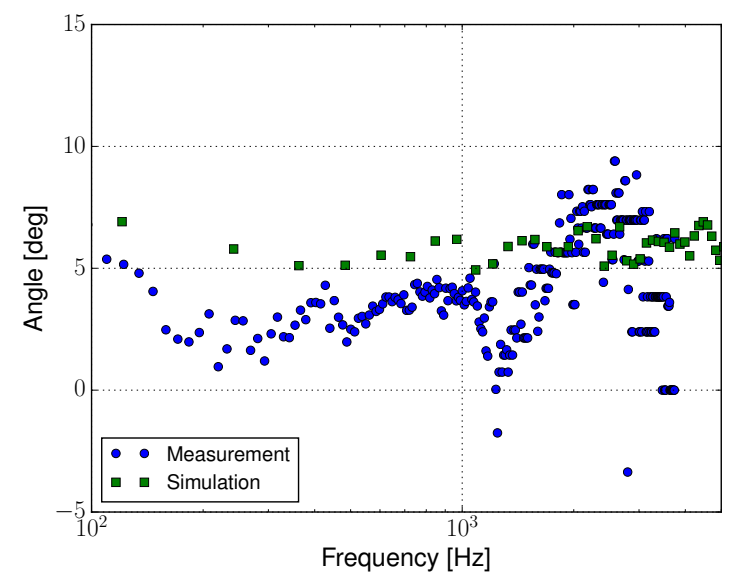

(a)

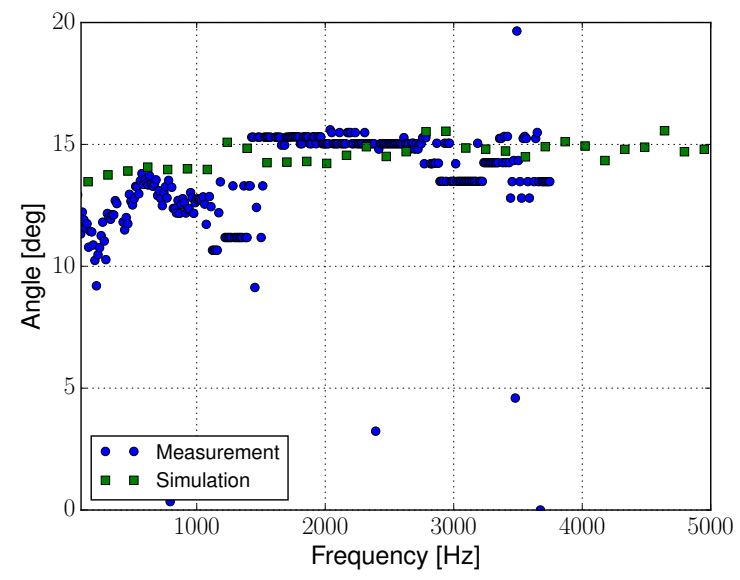

(b)

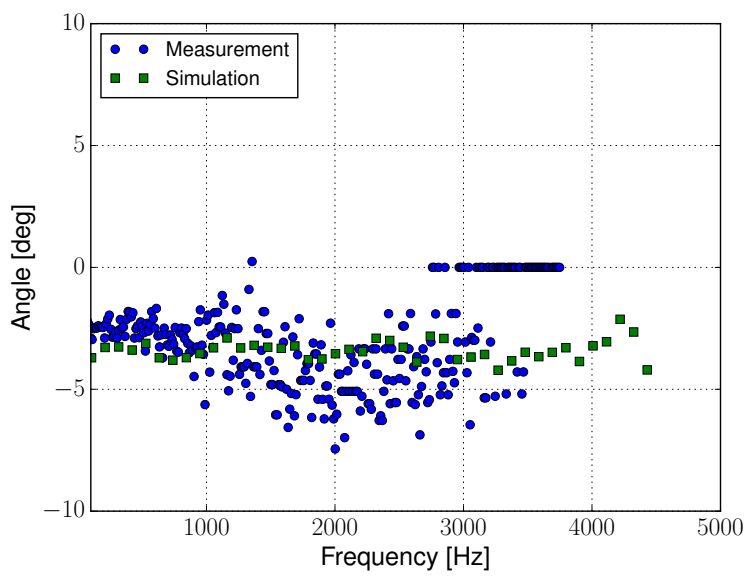

(c)

Fig. 7 Convection angles at different streamwise positions, (a) front; (b) mid; (c) aft.

reaches $15^{\circ}$. Downstream of the wing area, the flow deviates to a small negative inclination, which results in a small negative convection angle at the aft position.

Since the convection angle is available, the coherence lengths can be evaluated by fitting the rhombic formulation Eq. (23) or the elliptic formulation Eq. (24) to the simulated and measured coherence. For the fittings, the absolute value of the coherence from Eqs. $23,24 \mathrm{p}$ is used. Figs. 8 -9 show the obtained streamwise and spanwise coherence lengths for the calculated positions. The discrete deviations at multiples of $400 \mathrm{~Hz}$ shown in the measured results are caused by electrical noise. The coherence lengths derived from the rhombic shaped coherence field are larger than those from the elliptical shaped field. Due to the flow convection, the streamwise coherence length is much larger than the spanwise length. However, the spectral shape of the coherence lengths in both directions is similar. Those features are represented in both measurement and simulation results. 


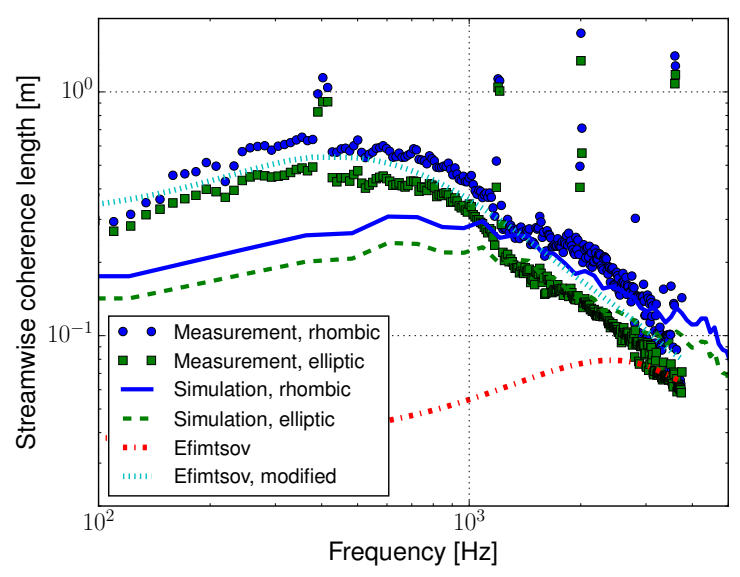

(a)

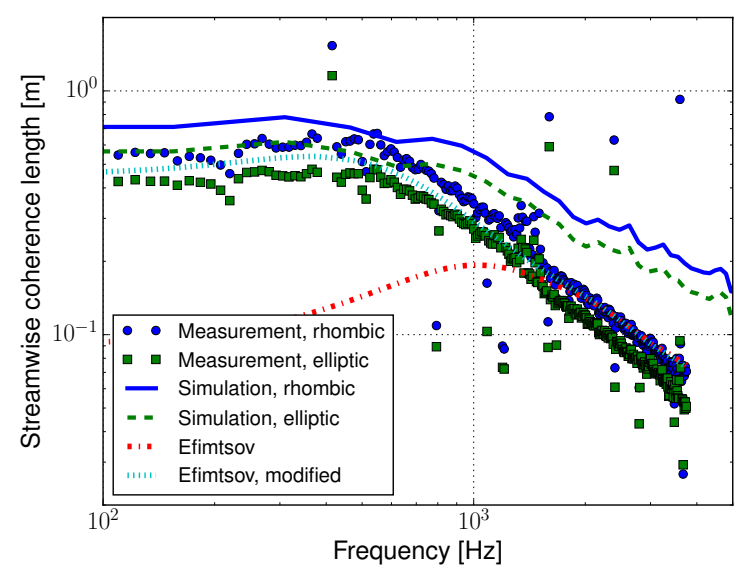

(b)

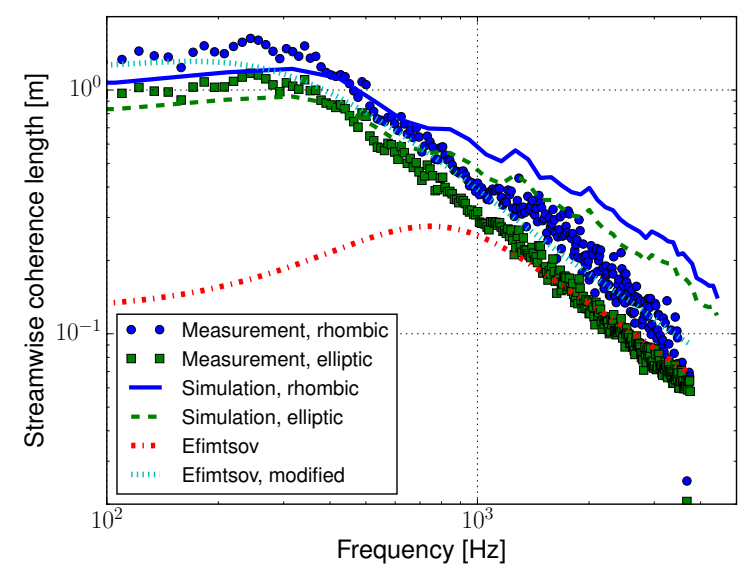

(c)

Fig. 8 Streamwise coherence lengths at different streamwise positions, (a) front; (b) mid; (c) aft.

Fig. 8 shows that the simulated streamwise coherence lengths are in good agreement with the measurement results at the mid and aft positions. However, for the front position the simulated magnitude of the coherence lengths is too small at low frequencies compared to the measurement results. The reason for that is not clear to the authors. Furthermore, the simulation results show a flatter spectral slope at high frequencies compared to the measurement results for all the positions. This indicates that the simulated coherence decay is slower at higher frequencies. One possible reason for that is the lack of the realized turbulence velocity fluctuations in the near wall area, refer to Fig. 3 (a). The high-frequency surface pressure fluctuations are mostly contributed by the small eddies close to the wall. However, due to the lack of contribution from the near wall area, the larger eddies farther away from the wall gain importance at high frequencies. Those larger eddies, due to their larger size and faster convection velocity, die out over a larger distance, which increases the simulated streamwise coherence length at high frequencies. 


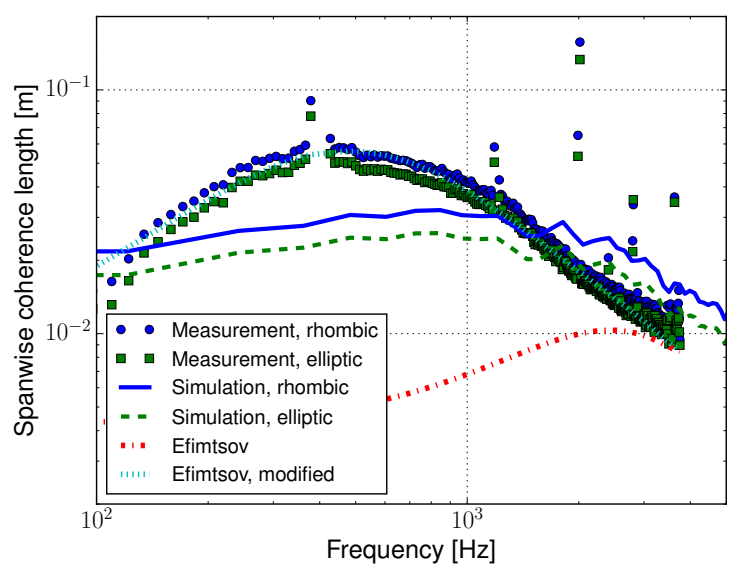

(a)

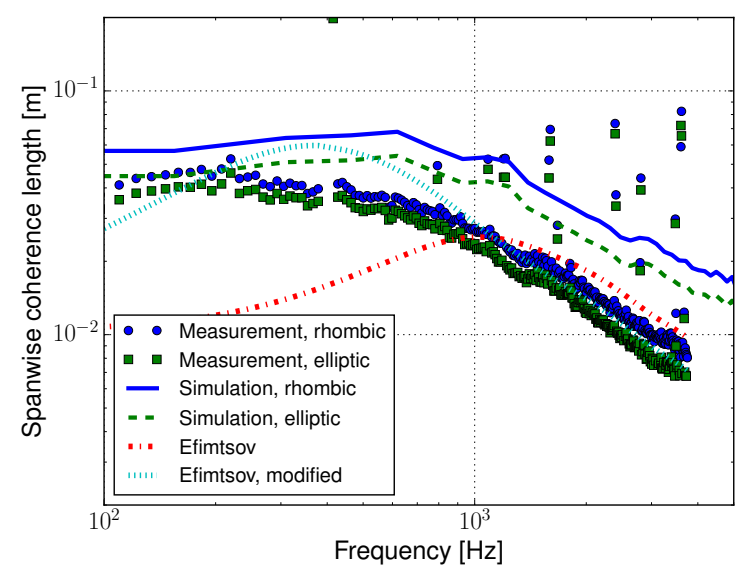

(b)

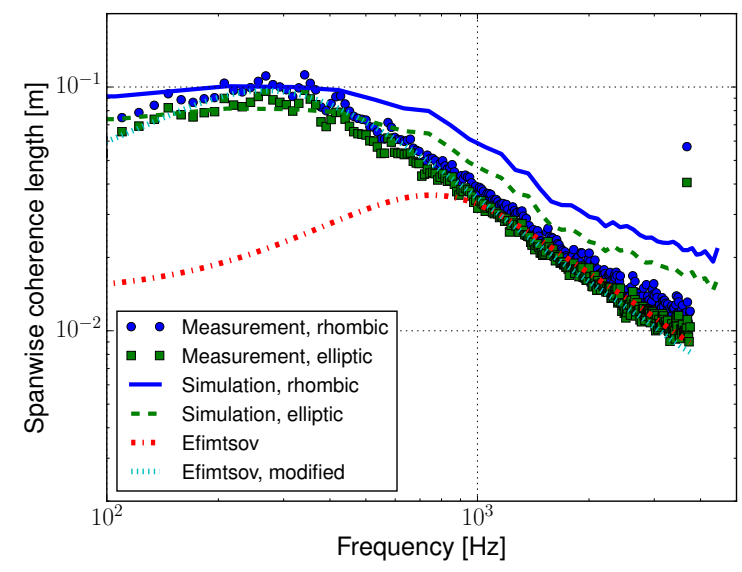

(c)

Fig. 9 Spanwise coherence lengths at different streamwise positions, (a) front; (b) mid; (c) aft.

Similar to the streamwise direction, the simulated spanwise coherence length is smaller at low frequencies compared to the measurement results for the front position, see Fig. 9. At the aft position, good agreement between the measured and simulated results is found. However, the simulated spanwise coherence length at the mid position is considerably larger than the measured results. This problem is probably related to the presence of the wing, which compresses streamlines in direction normal to the wing corresponding to the spanwise direction for the fuselage. The streamline compression will shorten the spanwise coherence length and this effect cannot be covered by the present numerical approach using a constant stretching factor for the realization of the turbulence anisotropy.

Furthermore, Figs. 86 9 show comparisons between the Efimtsov model [38] and the measured and simulated coherence lengths. The Efimtsov model was developed based on a series of flight tests and took into account the 
Table 1 Original and modified parameters of the Efimtsov model [38] for the coherence length.

\begin{tabular}{ccccccc} 
& $a_{1}$ & $a_{2}$ & $a_{3}$ & $a_{1}$ & $a_{2}$ & $a_{3}$ \\
& \multicolumn{3}{c}{ Streamwise } & & \multicolumn{3}{c}{ Spanwise } \\
\hline Original & 0.1 & 72.8 & 1.54 & 0.77 & 548 & 13.5 \\
Front & 0.09 & 1.73 & 0.19 & 0.83 & 16.3 & 10.3 \\
Mid & 0.1 & 11.3 & 0.31 & 1.05 & 70 & 10.6 \\
Aft & 0.08 & 5.47 & 0.16 & 0.84 & 68.7 & 5.5
\end{tabular}

low-frequency limitation of the coherence length, expressed as

$$
l_{1,3}(\omega)=\delta\left[\left(\frac{a_{1} \mathrm{Sh}}{U_{c} / u_{\tau}}\right)^{2}+\frac{a_{2}^{2}}{\mathrm{Sh}^{2}+\left(a_{2} / a_{3}\right)^{2}}\right]^{-1 / 2},
$$

where $\mathrm{Sh}=\omega \delta / u_{\tau}$, and the parameters $a_{1,2,3}$ determine the feature of the coherence length. The magnitude of the coherence length spectrum is adjusted by $a_{1}$. The parameter $a_{2}$ controls the peak position. The larger the value of $a_{2}$ is, the higher the predicted peak frequency is. The ratio of $a_{2} / a_{3}$ affects the low-frequency spectral shape. A reduced ratio will steepen the low-frequency spectral slope. The values of these parameters are listed in Table 1 along with the modified parameters, with which the best fitting to the measurement results is achieved.

With the original parameters, the peak frequency is predicted too high for all the positions and for both streamwise and spanwise directions. The reason for this is due to the excessively large value of $a_{2}$ suggested by Efimtsov [38], refer to Table 1. For frequencies above the peak, the prediction shows an excellent agreement with the measured results except for the spanwise coherence length at the mid position, where an overprediction of the magnitude can be observed. For this case, the numerical result and even the prediction with the modified parameters are not satisfactory. Note, that an upper limit of $a_{1,2}$ is made during the fitting process for this case because no clear peak is shown in the spectrum. The unsatisfactory result herein is probably due to the complicated flow condition caused by the airplane wing. For the other cases, an excellent agreement with the measured results can be achieved using the modified parameters listed in Table 1 The major modification of the parameters is that the value of $a_{2}$ is much smaller than the original one, enabling a good prediction of the peak frequency. This finding is in line with the results reported by Palumbo [16], Haxter and Spehr [18], Salze et al. [39]. Compared to the other positions, the modified value of $a_{2}$ is particularly small at the front position. Furthermore, the ratio of $a_{2} / a_{3}$ is also considerably smaller at the front position than that from the other positions and the original parameters, due to the significantly steeper low-frequency slope of the coherence length spectrum.

The convection velocity can be determined from the wavenumber-frequency spectra, which is calculated as $U_{c}(\omega)=\omega / \sqrt{k_{1}^{2}+k_{3}^{2}}$. The value of $k_{1,3}$ denotes the spectral peak position. Due to the flow convection, the peak of the convection ridge is located at the positive wavenumber $k_{1}$ domain, refer to Fig. 6 Fig. 10 shows the convection velocity derived from the numerical results and the predicted results using the Smol'yakov model [40] in comparison with the measured results. The numerical results show good accuracy of the spectral shape and magnitude for all three positions. 


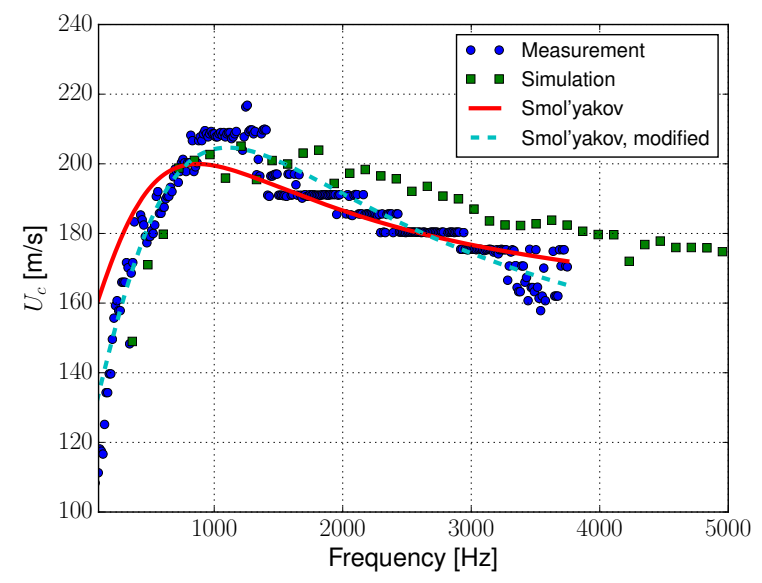

(a)

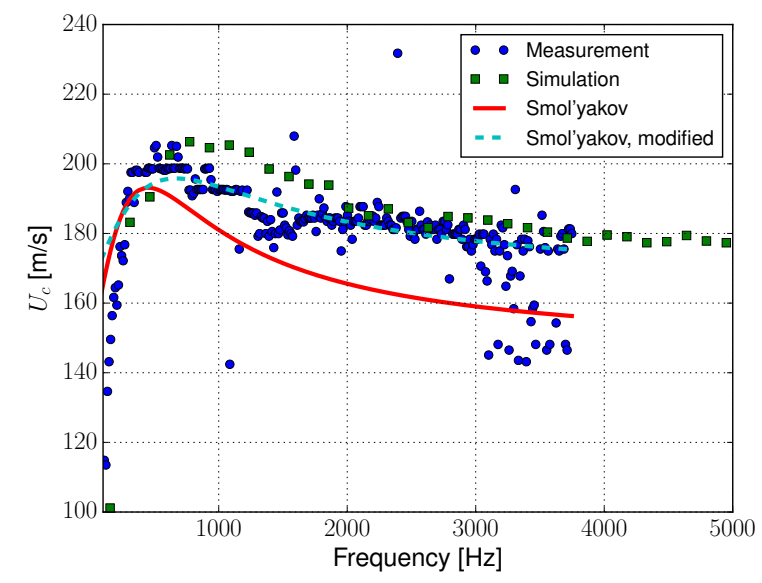

(b)

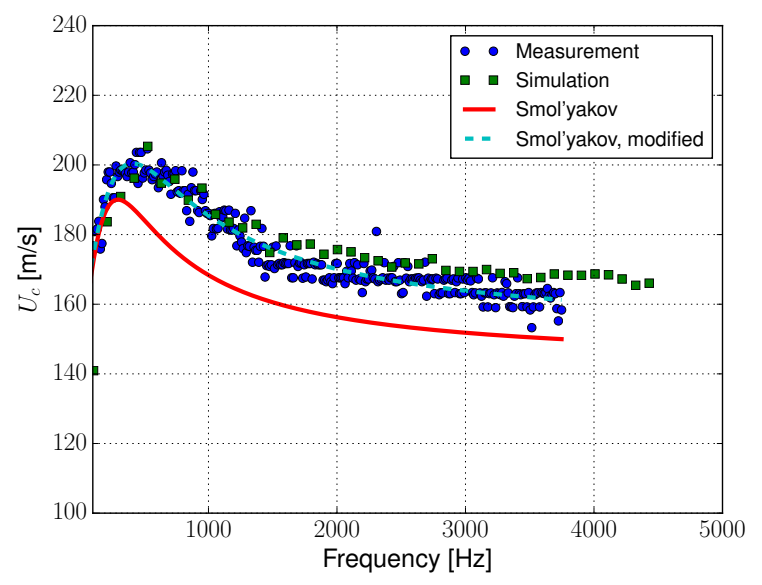

(c)

Fig. 10 Convection velocities at different streamwise positions, (a) front; (b) mid; (c) aft.

The velocity increases at low frequencies as the frequency increases. After reaching the maximum, the velocity falls down and approaches a nearly constant value at high frequencies. The Smol'yakov model, expressed as

$$
U_{c} / U_{0}=\frac{a_{1} \omega \delta^{*} / U_{0}}{1+a_{2}\left(\omega \delta^{*} / U_{0}\right)^{2}}+a_{3},
$$

gives a fairly good prediction of the frequency development of the convection velocity. However, in comparison with the measured results, a clear discrepancy of the magnitude with the original parameters (refer to Table 2 can be observed, e.g. an underprediction by about $10 \%$ at high frequencies for the mid and aft positions. With increased values of $a_{3}$ for these two positions, refer to Table 2, the velocity magnitude is well predicted. Furthermore, the modified values of $a_{1}$ are smaller than the original ones for these two positions with mild and slight favorable pressure gradients, respectively, refer to the Clauser parameter $\beta_{\delta^{*}}$ shown in Fig. 2. The modification of $a_{1,3}$ for the front position with a slight adverse 
Table 2 Original and modified parameters of the Smol'yakov model [40] for the convection velocity.

\begin{tabular}{cccc} 
& $a_{1}$ & $a_{2}$ & $a_{3}$ \\
\hline Original & 1.6 & 16 & 0.6 \\
Front & 2.18 & 9.95 & 0.47 \\
Mid & 0.7 & 7.28 & 0.68 \\
Aft & 1.22 & 8.37 & 0.63
\end{tabular}

pressure gradient shows an opposite trend, which may be a result of the pressure gradient effect. For all three positions, the modified value of $a_{2}$ is smaller than the original one. With this change, the predicted peak position can perfectly match the measured results.

\section{Conclusion}

Surface pressure fluctuations are simulated at three streamwise positions of an Airbus-A320 aircraft fuselage at cruise conditions. The computation is performed via the Poisson equation. Synthetic anisotropic turbulence is realized with the Fast Random Particle-Mesh Method, which is used to generate the source terms. Both mean-shear term and turbulence-turbulence term are considered. The mean flow statistics which are needed for the generation of the synthetic turbulence are obtained from a RANS calculation with DLR's in-house code TAU.

The simulation results for one-point spectra and two-point statistics are compared to the flight test results. The obtained spectra show good agreement with the measured results for all the three calculation positions until $5 \mathrm{kHz}$, which is the upper frequency range of interest to the industrial application. For the calculation of two-point statistics, a larger calculation area is required, and thus, a rougher mesh is applied. The rougher mesh worsens the realization of the small turbulence structures in the near wall area, which significantly lowers the one-point spectral magnitude at high frequencies and can also result in a reduction of the magnitude at low frequencies. A prediction of Goody's model [5] indicates that the spectral fall at high frequencies is significantly steeper than that for incompressible flows. This effect is probably due to the reduction of the air density close to the wall in compressible flows. An excellent prediction of the high-frequency spectral fall is provided by Hu's model [7], which manages the spectral slope with the help of the boundary layer shape factor. This may indicate that the effect of the density change on the surface pressure spectrum can be represented by the shape factor.

The coherence and wavenumber spectrum are obtained through a rectangular microphone array. The convection angle and velocity, the streamwise and spanwise coherence lengths are calculated and compared to the flight test results. The simulated results for the convection angle and velocity show very good agreement with the measured results for all three considered positions. The simulated spectra for the coherence lengths reproduce well the measured spectral trends. Exceptions are the low-frequency magnitude for both streamwise and spanwise coherence lengths at the front position and the magnitude for the spanwise coherence length at the mid position. For the latter case, even Efimtsov's model [38] 


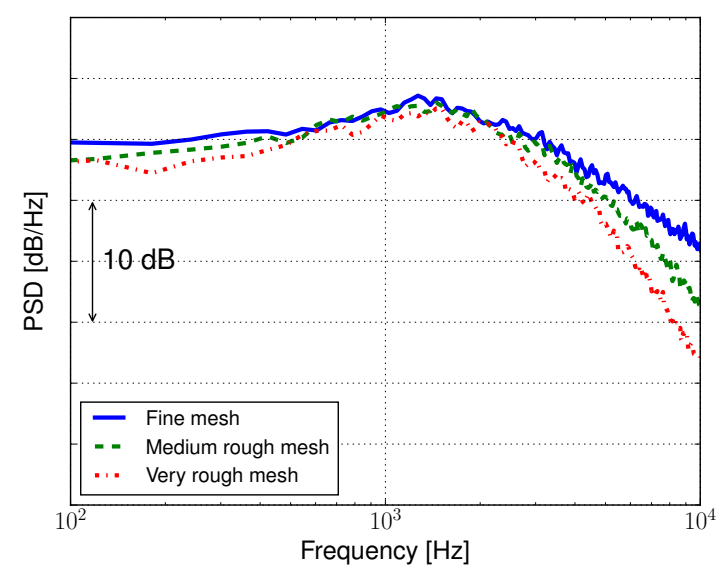

(a)

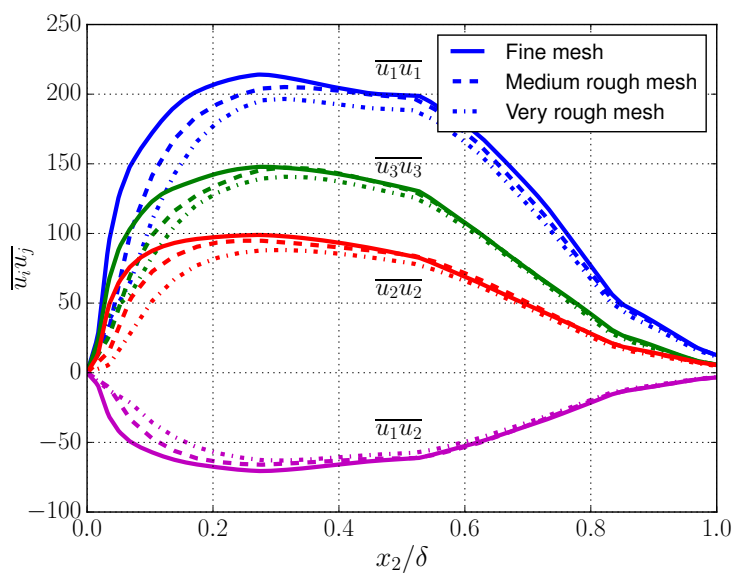

(b)

Fig. 11 (a) One-point spectra; (b) Reconstruction of Reynolds stress components.

with the best-fit parameter values cannot produce a good prediction. This unsatisfactory result is probably due to the complicated flow condition in the vicinity of the wing.

The simulation results confirm that the surface pressure fluctuations beneath compressible boundary layer flows under cruise conditions can be calculated with the Poisson equation. Features of the fuselage surface pressure fluctuations relevant to the surface excitation can be well reproduced. The obtained results can be used for the further calculation of the fuselage vibration.

\section{A. Calculations with different mesh resolutions}

Fig. 11 shows the calculated surface pressure spectra and the reconstruction of Reynolds stress components by the FRPM realization for the front position. The calculations are conducted in the small computational domain described in Sec. [III.B The fine mesh denotes the same mesh as used in the main text, with 128 points in the streamwise direction, 64 points in the wall-normal direction and 128 points in the spanwise direction. The mesh points of the medium rough mesh are halved in each direction, i.e. $64 \times 32 \times 64$ points. For the very rough mesh, there are 32 points in each direction.

Due to the rough mesh resolution, the lack of the Reynolds stress reconstruction can be observed mainly in the region $<0.3 \delta$ and this problem is more severe with increasing mesh roughness. Consequently, a significant spectral attenuation at high frequencies is shown for the rough mesh cases in comparison with the fine mesh. Furthermore, an attenuation at low frequencies is also caused, which is larger with increasing mesh roughness.

\section{Acknowledgments}

This work was supported through joint funding of DLR and Airbus. The authors would like to thank the DLR colleague Dr.-Ing. B. Eisfeld for the advice on the transformation of the Reynolds stress. 


\section{References}

[1] Hu, N., Buchholz, H., Herr, M., Spehr, C., and Haxter, S., "Contributions of Different Aeroacoustic Sources to Aircraft Cabin Noise," AIAA Paper 2013-2030, 2013. doi:10.2514/6.2013-2030.

[2] Willmarth, W. W., and Wooldridge, C. E., "Measurements of the fluctuating pressure at the wall beneath a thick turbulent boundary layer,” J. Fluid Mech., Vol. 14, 1962, pp. 187-210. doi:10.1017/S0022112062001160.

[3] Farabee, T. M., and Casarella, M. J., "Spectral features of wall pressure fluctuations beneath turbulent boundary layers," Phys. Fluids, Vol. A3(10), 1991, pp. 2410-2420. doi:10.1063/1.858179.

[4] Corcos, G. M., “The Structure of the Turbulent Pressure Field in Boundary Layer Flows,” J. Fluid Mech., Vol. 18, 1964, pp. 353-378. doi:10.1017/S002211206400026X.

[5] Goody, M. C., "Empirical Spectral Model of Surface Pressure Fluctuations," AIAA Journal, Vol. 42(9), 2004, pp. 1788-1794. doi:10.2514/1.9433.

[6] Rozenberg, Y., Robert, G., and Moreau, S., "Wall-pressure spectral model including the adverse pressure gradient effects," AIAA Journal, Vol. 50(10), 2012, pp. 2168-2179. doi:10.2514/1.J051500.

[7] Hu, N., "Empirical model of wall pressure spectra in adverse pressure gradients," AIAA Journal, Vol. 56(9), 2018, pp. 3491-3506. doi:10.2514/1.J056666.

[8] Kim, J., "On the structure of pressure fluctuations in simulated turbulent channel flow," J. Fluid Mech., Vol. 205, 1989, pp. 421-451. doi:10.1017/S0022112089002090.

[9] Chang, P., Piomelli, U., and Blake, W. K., "Relationship between wall pressure and velocity-field sources," Phys. Fluids, Vol. 11, 1999, pp. 3434-3448. doi:10.1063/1.870202.

[10] Ehrenfried, K., and Koop, L., "Experimental study of pressure fluctuations beneath a compressible turbulent boundary layer," AIAA Paper 2008-2800, 2008. doi:10.2514/6.2008-2800.

[11] Gloerfelt, X., and Berland, J., “Turbulent boundary-layer noise: direct radiation at Mach number 0.5," J. Fluid Mech., Vol. 723, 2013, pp. 318-351. doi:10.1017/jfm.2013.134.

[12] Duan, L., Choudhari, M. M., and Wu, M., "Numerical study of acoustic radiation due to a supersonic turbulent boundary layer," J. Fluid Mech, Vol. 746, 2014, pp. 165-192. doi:10.1017/jfm.2014.116.

[13] Cohen, E., and Gloerfeltc, X., "Influence of pressure gradients on wall pressure beneath a turbulent boundary layer," J. Fluid Mech., Vol. 838, 2018, pp. 715-758. doi:10.1017/jfm.2017.898.

[14] Bhat, W. V., "Flight Test Measurement of Exterior Turbulent Boundary Layer Pressure Fluctuations on Boeing Model 737 Airplane,” J. Sound Vib., Vol. 14(4), 1971, pp. 439-457. doi:10.1016/0022-460X(71)90574-8. 
[15] Palumbo, D., "Deriving Lifetime Maps of Coherent Structures in the Turbulent Boundary Layer," AIAA Journal, Vol. 46(4), 2008, pp. 810-823. doi:10.2514/1.30644.

[16] Palumbo, D., "Determining correlation and coherence lengths in turbulent boundary layer flight data," J. Sound Vib., Vol. 331, 2012, pp. 3721-3737. doi:10.1016/j.jsv.2012.03.015.

[17] Haxter, S., and Spehr, C., "Two-Dimensional Evaluation of Turbulent Boundary Layer Pressure Fluctuations at Cruise Flight Conditions," AIAA Paper 2012-2139, 2012. doi:10.2514/6.2012-2139.

[18] Haxter, S., and Spehr, C., "Comparison of model predictions for coherence length to in-flight measurements at cruise conditions," J. Sound Vib., Vol. 390, 2017, pp. 86-117. doi:10.1016/j.jsv.2016.10.038.

[19] Klabes, A., Appel, C., Herr, M., and Bouhaj, M., "Fuselage excitation during cruise flight conditions: measurement and prediction of pressure point spectra," AIAA Paper 2015-3115, 2015. doi:10.2514/6.2015-3115.

[20] Spehr, C., Hennings, H., Buchholz, H., Bouhaj, M., Haxter, S., and Hebler, A., "In-flight sound Measurements: A first overview," AIAA Paper 2012-2208, 2012. doi:10.2514/6.2012-2208.

[21] Hu, N., Reiche, N., and Ewert, R., "Simulation of turbulent boundary layer wall pressure fluctuations via Poisson equation and synthetic turbulence,” J. Fluid Mech., Vol. 826, 2017, pp. 421-454. doi:10.1017/jfm.2017.448.

[22] Ewert, R., Dierke, J., Siebert, J., Neifeld, A., Appel, C., Siefert, M., and Kornow, O., “CAA broadband noise prediction for aeroacoustic design,” J. Sound Vib., Vol. 330, 2011, pp. 4139-4160. doi:10.1016/j.jsv.2011.04.014.

[23] Haxter, S., and Spehr, C., "Up in the Air: In-Flight Wavenumber Characterization of Surface Pressure Fluctuations at Transonic Conditions," AIAA Paper 2018-3275, 2018. doi:10.2514/6.2018-3275.

[24] Hockney, R. W., and Eastwood, J. W., Computer simulation using particles, Taylor \& Francis, Inc., 1988.

[25] Bailly, C., and Juvé, D., “A stochastic approach to compute subsonic noise using linearized Euler's equations,” AIAA Paper 1999-1872, 1999. doi:10.2514/6.1999-1872.

[26] Schwamborn, D., Gerhold, T., and Kessler, R., "DLR-TAU Code - an overview," 1st ONERA/DLR Aerospace Symposium, Paris, 1999.

[27] Togiti, V., and Eisfeld, B., "Assessment of g-equation formulation for a second-moment Reynolds stress turbulence model," AIAA Paper 2015-2925, 2015. doi:10.2514/6.2015-2925.

[28] Leatham, M., Stokes, S., Shaw, J. A., Cooper, J., Appa, J., and Blaylock, T. A., "Automatic mesh generation for rapid-response Navier-Stokes calculations," AIAA Paper 2000-2247, 2000. doi:10.2514/6.2000-2247.

[29] Klabes, A., "Aircraft fuselage vibration excitation by turbulent boundary layer flow in cruise," Ph.D. thesis, Institute of Aerodynamics and Flow Technology, German Aerospace Center, 2017. 
[30] Nagib, H. M., Chauhan, K. A., and Monkewitz, P. A., "Approach to an asymptotic state for zero pressure gradient turbulent boundary layers," Phil. Trans. R. Soc. A, Vol. 365, 2007, pp. 755-770. doi:10.1098/rsta.2006.1948.

[31] Gerolymos, G. A., and Vallet, I., "Pressure, density, temperature and entropy fluctuations in compressible turbulent plane channel flow," J. Fluid Mech., Vol. 757, 2014, pp. 701-746. doi:10.1017/jfm.2014.431.

[32] Welch, P. D., "The use of fast Fourier transform for the estimation of power spectra: A method based on time averaging over short, modified periodograms," IEEE Transactions on Audio and Electroacoustics, Vol. 15 (2), 1967, pp. 70-73. doi:10.1109/TAU.1967.1161901.

[33] Panton, R. L., and Linebarger, J. H., "Wall pressure spectra for equilibrium boundary layers," J. Fluid Mech., Vol. 65, 1974, pp. 261-287. doi:10.1017/S0022112074001388.

[34] Blake, W. K., Mechanics of flow-induced sound and vibration, Academic Press, Inc., 1986.

[35] Hu, N., and Callsen, S., "A parametric sutdy on wall pressure wavenumberspectrum models with application to aircraft fuselage vibration prediction," 26th International Congress on Sound and Vibration, 2019.

[36] Smol'yakov, A., and Tkachenko, V., "Model of a field of pseudosonic turbulent wall pressures and experimental data," Akust. Zh., Vol. 37, 1991, pp. 1199-1207.

[37] Haxter, S., Berkefeld, T., and Spehr, C., "Determining Flow Propagation Direction from In-Flight Array Surface Pressure Fluctuation Data," AIAA Paper 2017-3205, 2017. doi:10.2514/6.2017-3205.

[38] Efimtsov, B., "Characteristics of the field of turbulent wall pressure fluctuations at large Reynolds numbers," Sov. Phys. Acoust, Vol. 28, 1982, pp. 289-292.

[39] Salze, E., Bailly, C., Marsden, O., Jondeau, E., and Juvé, D., “An experimental characterisation of wall pressure wavevectorfrequency spectra in the presence of pressure gradients," AIAA Paper 2014-2909, 2014. doi:10.2514/6.2014-2909.

[40] Smol'yakov, A., "A new model for the cross spectrum and wavenumber-frequency spectrum of turbulent pressure fluctuations in a boundary layer," Acoustical Physics, Vol. 52(3), 2006, pp. 331-337. doi:10.1134/S1063771006030146. 